\title{
Facial Flap Repositioning in Posttraumatic Facial Asymmetry
}

\author{
Il Hwan Byun, \\ Dahn Byun', \\ Woo Yeol Baek ${ }^{1}$ \\ ${ }^{1}$ Department of Plastic and Reconstructive \\ Surgery, Yonsei University College of Medicine, \\ Seoul; \\ ${ }^{2}$ Eulji University College of Medicine, Daejeon, \\ Korea
}

No potential conflict of interest relevant to this article was reported.

\begin{abstract}
Perfect facial and body symmetry is an important aesthetic concept which is very difficult, if not impossible, to achieve. Yet, facial asymmetries are commonly encountered by plastic and reconstructive surgeons. Here, we present a case of posttraumatic facial asymmetry successfully treated with a unique concept of facial flap repositioning. A 25 -year-old male patient visited our department with severe posttraumatic facial asymmetry. There was deviated nasal bone and implant to the right, and the actual facial appearance asymmetry was much more severe compared to the computed tomography, generally shifted to the right. After corrective rhinoplasty, we approached through intraoral incision, and much adhesion from previous surgeries was noted. We meticulously elevated the facial flap of both sides, mainly involving the cheeks. The elevated facial flap was shifted to the left, and after finding the appropriate location, we sutured the middle portion of the flap to the periosteum of anterior nasal spine for fixation. We successfully freed the deviated facial tissues and repositioned it to improve symmetry in a single stage operation. We conclude that facial flap repositioning is an effective technique for patients with multiple operation history, and such method can successfully apply to other body parts with decreased tissue laxity.
\end{abstract}

Keywords: Facial asymmetry / Surgical flap

\section{INTRODUCTION}

Perfect facial and body symmetry is an important aesthetic concept which is very difficult, if not impossible, to achieve. Morphological differences occur in every living organism where two bilateral congruent parts are present [1]. Functional asymmetries exist as well, including right and left handedness and eye preference. Genetics and embryologymay contribute to such asymmetry, but some are idiopathic and unclear. Regarding facial asymmetry, previous studies classified the etiology into three categories: congenital, developmental, and acquired (Table 1) [2,3]. Both congenital and acquired facial asymmetries are commonly encountered

\section{Correspondence: Woo Yeol Baek}

Department of Plastic and Reconstructive Surgery, Yonsei University College of Medicine, 50-1 Yonsei-ro, Seodaemun-gu, Seoul 03722, Korea

E-mail: parande@yuhs.ac

Received September 18, 2016 / Revised December 14, 2016 / Accepted December 14, 2016 by plastic and reconstructive surgeons.

Though minor facial asymmetry is common and indiscernible, severe asymmetry that exceeds sense of normal balance noted by the clinician and the patient often requires treatment [4]. Here, we present a case of posttraumatic facial asymmetry successfully treated with a unique concept of facial flap repositioning. Although most posttraumatic facial deformities involve facial

Table 1. Etiology of facial asymmetry

\section{Variable}

Congenital Cleft lip and palate, Tessier craniofacial cleft, hemifacial microsomia, neurofibromatosis, torticollis, craniosynostosis, vascular disorders, etc.

\begin{tabular}{ll} 
Develpmental & Unknown cause \\
Acquired & $\begin{array}{c}\text { Temporomandibular joint ankylosis, facial trauma, } \\
\text { radiotherapy, fibrous dysplasia, facial tumors, } \\
\text { unilateral condylar hyperplasia, Romberg's } \\
\text { disease, etc. }\end{array}$ \\
\hline
\end{tabular}


bone fractures, our patient developed severe asymmetry even after multiple operations of the facial bones.

\section{CASE REPORT}

A 25-year-old male patient visited our department with severe posttraumatic facial asymmetry. He went through a vicious traffic accident, leading to a panfacial fracture six years ago. He had bilateral inferior orbital rim and zygomaticomaxillary fractures at the time and received over four surgeries over several years in other hospitals. The operations included open reduction, one jaw surgery with Le Fort I osteotomy, corrective rhinoplasty, and augmentation rhinoplasty. Three-dimensional computed tomography (CT) was taken to evaluate facial bone status (Fig. 1). There was deviated nasal bone and implant to the right, yet other bones were nearly symmetrical. However, the actual facial appearance asymmetry was much more severe compared to the bone CT (Fig. 2). The face was generally shifted to the right. Adhesion and contracture from previous operations seemed present, and the patient requested treatment of the prominent appearing asymmetry.

Under general anesthesia, the patient was placed on the operative table in supine position. First for the deviated nose and flared nostrils, we performed open rhinoplasty involving implant change, columella strut formation, nasal tip plasty, onlay graft, and nostril sil reduction. This improved the nasal appearance, and the next major problem was deviated facial skin. We ap-

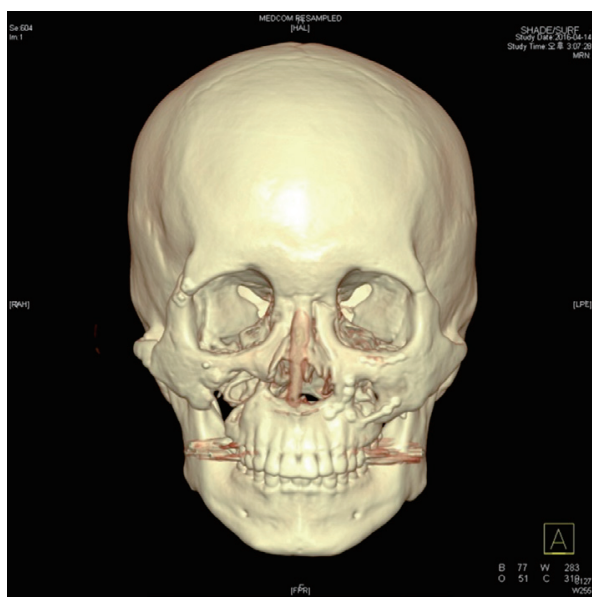

Fig. 1. Preoperative 3-dimensional computed tomography. proached through a $5 \mathrm{~cm}$ intraoral incision on the upper gingiva, and much adhesion from previous surgeries was noted as expected. We meticulously undermined and elevated the facial flap of both sides, mainly involving the cheeks (Fig. 2). No nerves were damaged. The elevated facial flap was shifted to the left, and after finding the appropriate location, we sutured the middle portion of the flap to the periosteum of anterior nasal spine for fixation. Not too many sutures were done considering natural facial expressions in the future. One Silastic drain was inserted to the intraoral incision.

Daily simple dressing was done postoperatively, and the drain was removed two days after the surgery. Nasal stitches were removed one week after the operation. The incision sites remained clean, and no dehiscence, infection, hematoma, or seroma developed. No complication such as nerve injury was noted, and the patient was pleased with the result. The general appearance of the face improved greatly with better symmetry, and the nasal deviation improved (Fig. 3). To evaluate objective outcome of the operation, we calculated two angles of the face. First we calculated the change in angle of both lateral commissures. The horizontal reference line was set between bilateral soft tissue gonions. Second, we drew an imaginary line between the glabella and upper stomion, and measured the change in deviation from the midsagittal line.

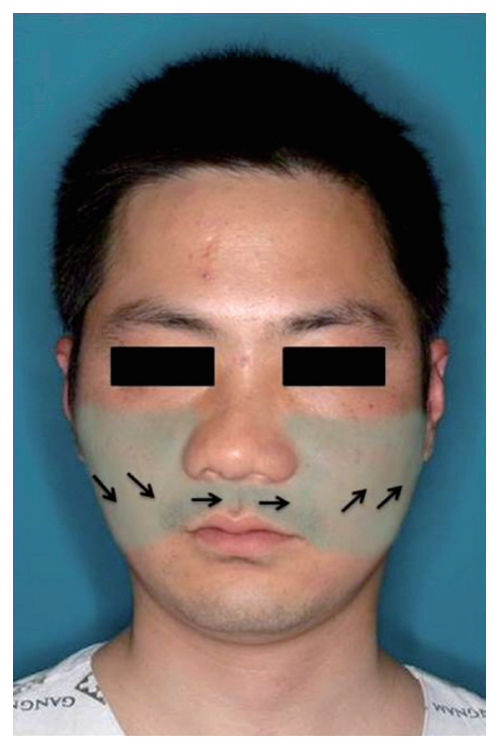

Fig. 2. Preoperative anterior view with the areas of dissection shaded in light green. 

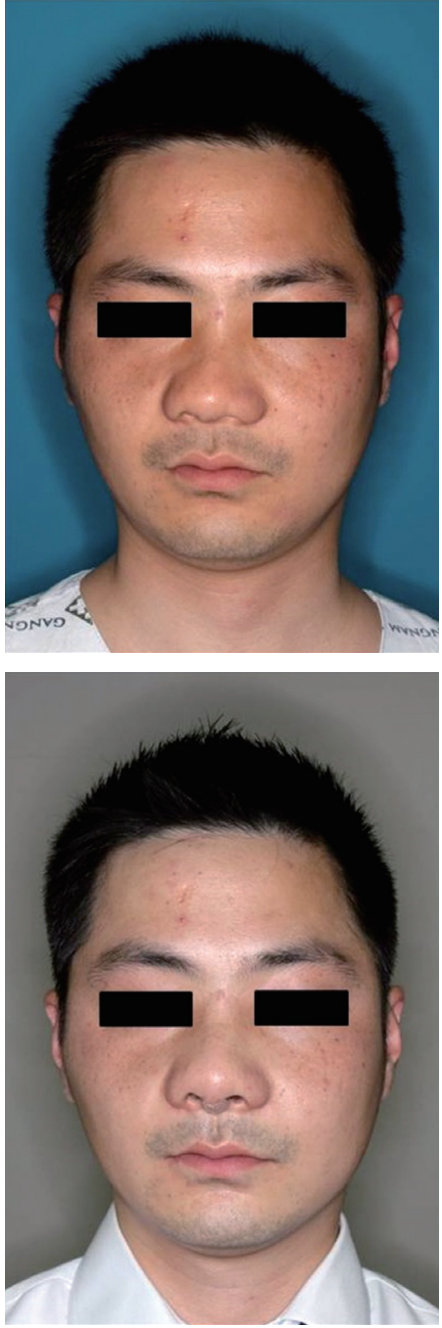

Fig. 3. Preoperative and postoperative 2 week view.

The horizontal reference line was set between bilateral external canthi. We set the midsagittal line as the vertical line passing through the center of this horizontal line. The first angle of lateral commissures improved from $4.24^{\circ}$ to $1.27^{\circ}$, and the second angle of vertical deviation decreased from $3.96^{\circ}$ to $1.12^{\circ}$ in two week follow-up (Fig. 4). Even after six months, the postoperative result was generally maintained (Fig. 5).

\section{DISCUSSION}

Facial asymmetry is a commonly encountered condition in the field of plastic and reconstructive surgery, and various surgical
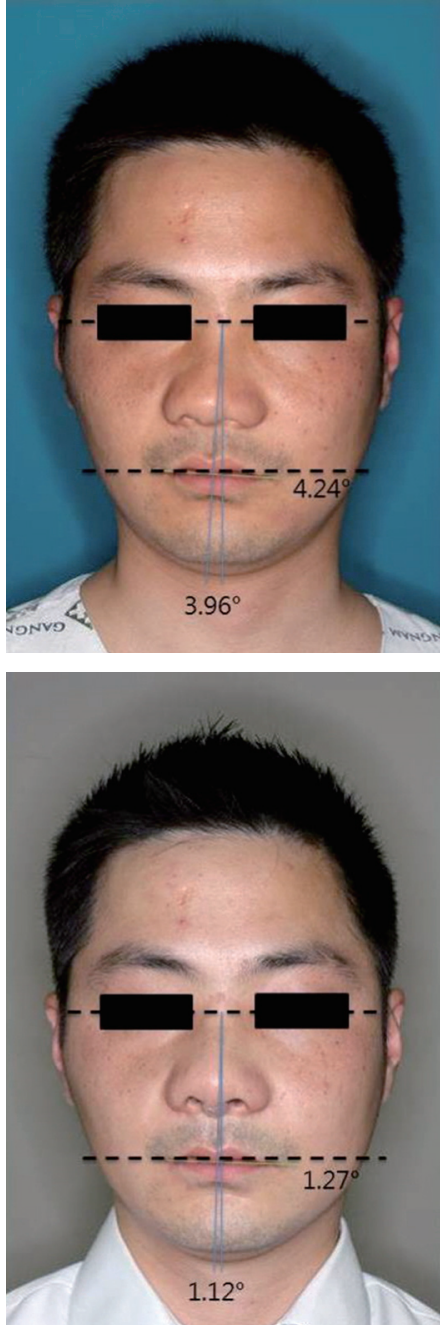

Fig. 4. Angles of preoperative and postoperative facial deviation.

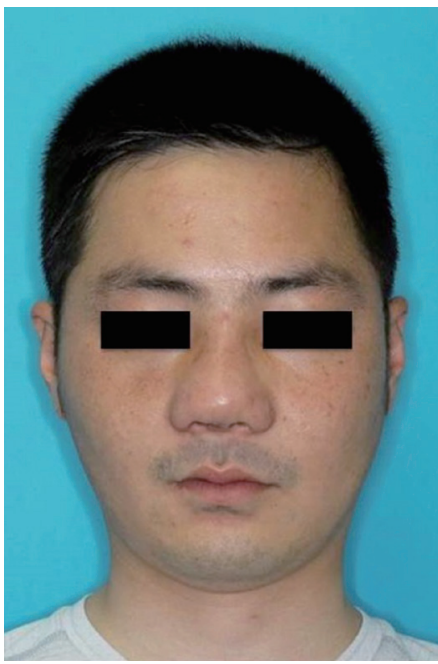

Fig. 5. Postoperative 6 month view. 
options exist. Severe facial asymmetry is known to cause psychological and emotional distress and may contribute to functional impairment as well [5]. As with any other patients, thorough evaluation including history taking, physical examination, and imaging studies is crucial in treating facial asymmetry patients [1]. Facial asymmetry involves skeletal, dental, and soft tissue components, and such factors as well as patient perceptions and expectations must be carefully considered [1].

Traditional surgical techniques include Le Fort I osteotomy, bilateral sagittal split osteotomy, and two-jaw orthognathic surgery. Recently, combination of various techniques such as bone contouring and fat graft injection prevailed and led to aesthetic results. Choi et al. [6] reported bimaxillary orthognathic surgery combined with a face lift procedure plus the use of a resorbable fixation device. In this case, we judged that the patient did not require more facial bone surgery, yet face lift or fat graft injection did not seem sufficient to correct the asymmetry. Thus we proceeded with facial repositioning along with corrective rhinoplasty, and the calculated angles show numerical proof of the good result.

The result of the operation is satisfactory, yet this case has a limitation of the follow-up period. This patient has received several operations before this one, and he may develop adhesion and partial regression in the future. Long-term follow-up and evaluation of more similar cases are required in the future.

This is a meaningful case applying the concept of facial flap repositioning. Our patient received multiple operations on facial bone fracture, yet his asymmetry worsened as the soft tissue deviated with adhesion and contraction. Along with corrective rhinoplasty for nasal deviation, we successfully freed the deviated facial tissues and repositioned it to improve symmetry in a single stage operation. The patient developed no motor or sensory complications. We conclude that facial flap repositioning is an effective technique for patients with multiple operation history, and such method can successfully apply to other body parts with decreased tissue laxity.

\section{REFERENCES}

1. Cheong YW, Lo LJ. Facial asymmetry: etiology, evaluation, and management. Chang Gung Med J 2011;34:341-51.

2. Cohen MM Jr. Perspectives on craniofacial asymmetry: III. Common and/or well-known causes of asymmetry. Int J Oral Maxillofac Surg 1995;24:127-33.

3. Reyneke JP, Tsakiris P, Kienle F. A simple classification for surgical treatment planning of maxillomandibular asymmetry. Br J Oral Maxillofac Surg 1997;35:349-51.

4. Bishara SE, Burkey PS, Kharouf JG. Dental and facial asymmetries: a review. Angle Orthod 1994;64:89-98

5. Shackelford TK, Larsen RJ. Facial asymmetry as an indicator of psychological, emotional, and physiological distress. J Pers Soc Psychol 1997;72:456-66.

6. Choi JY, Choi JP, Lee YK, Baek SH. Simultaneous correction of hardand soft-tissue facial asymmetry: combination of orthognathic surgery and face lift using a resorbable fixation device. J Craniofac Surg 2010;21:363-70. 